\title{
Wiskott-Aldrich Syndrome
}

National Cancer Institute

\section{Source}

National Cancer Institute. Wiskott-Aldrich Syndrome. NCI Thesaurus. Code C3448.

A rare, X-linked immunodeficiency syndrome characterized by eczema,

thrombocytopenia, and recurrent pyogenic infection. It is seen exclusively in young boys.

Typically, Ig M levels are low and IgA and IgE levels are elevated. Lymphoreticular malignancies are common. 